\title{
Subwavelength Focusing of Light without Evanescent Waves by an Array of Nanoholes
}

\author{
Fu Min Huang ${ }^{1}$, Yifang Chen ${ }^{2}$, F. Javier Garcia de Abajo ${ }^{3}$ and Nikolay Zheludev ${ }^{1}$ \\ ${ }^{I}$ EPSRC Nanophotonics Portfolio Centre, Optoelectronics Research Center, University of Southampton, SO17 1BJ, UK \\ ${ }^{2}$ Central Microstructure facility, Rutherford Appleton Laboratory, Didcot, Oxon, OX11 OQX, UK \\ ${ }^{3}$ Instituto de Optica, CSIC, Serrano 121, 28006 Madrid, Spain \\ Email:fmh@orc.soton.ac.uk
}

\begin{abstract}
We provide the first evidence of free-space subwavelength focusing without evanescent fields using a photonic nano-structure. Hot-spots smaller than half of the wavelength were observed at distances of tens of wavelengths from the structure.

(C)2007 Optical Society of America

OCIS codes: (350.5730) Resolution; (070.2580) Fourier optics.
\end{abstract}

Here we provide the first experimental evidence of forming subwavelength fields without evanescent waves by diffraction on a 2-dimensional planar structure. A quasi-periodic array of nanoholes was observed to focus light into subwavelength spots in free space when illuminated with a coherent light source. Diffraction patterns with subwavelength spots were observed at distances of a few tens of wavelengths away from the nanohole array, both from a conventional optical microscope and a scanning near field optical microscope (SNOM). The ability to focus light into subwavelength spots is hugely important for a wide range of applications, e.g. microscopy, photo lithography, data storage etc.

Generally it was believed that the resolution of conventional optical microscopy is limited to about half wavelength of light while subwavelength focusing is only achievable in the proximity of a subwavelength aperture or nanostructures where evanescent waves exist. The limited resolution of far-field focusing results from the loss of evanescent fields in far field, which contain spatial frequencies larger than the wavevector of light and decay exponentially in free space. However, it was recently shown that evanescent waves are not the necessary conditions of forming subwavelength fields. Berry and Popescu demonstrated that functions with limited spectrum are able to oscillate much faster than the highest frequency components they contain, and that such oscillation may propagate in free space, a phenomenon they called superoscillations [1]. This suggests that light can form subwavelength fields without evanescent waves.
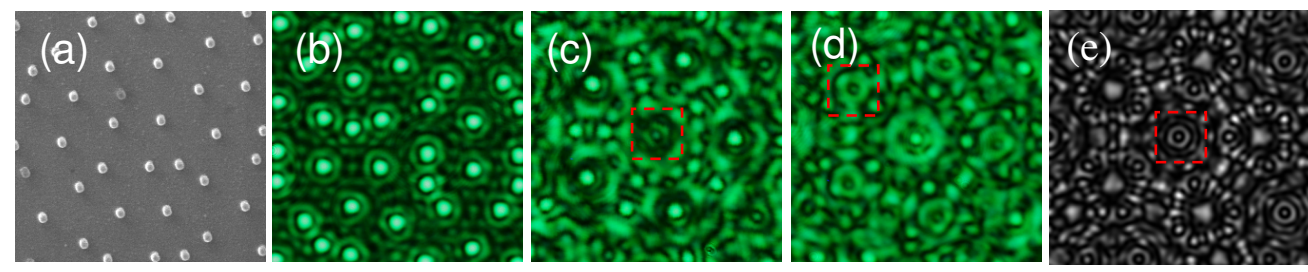

Fig.1 SEM (a) and photo (b) images of a fragment of the quasi-crystal sample. Photographs of the diffraction patterns at height $h=7 \mu \mathrm{m}$ (c) and $h=8.5 \mu \mathrm{m}$ (d). Two experimentally observed subwavelength spots with FWHM of $\sim 240 \mathrm{~nm}$ (c) and $\sim 170 \mathrm{~nm}$ (d) are highlighted. Figure (e) shows calculated field pattern similar to the experimental case (c) which indicates a hot spot with FWHM of $160 \mathrm{~nm}$ (marked central dot). The size of images (a-e) is $10 \mu \mathrm{m} \times 10 \mu \mathrm{m}, \lambda=$ $500 \mathrm{~nm}$.

In our experiments we used a Penrose-like quasi-crystal array of nanoholes with approximate 10-fold symmetry. It contains about 14,000 holes of $200 \mathrm{~nm}$ in diameter. The sample was manufactured by electron beam lithography in a $100 \mathrm{~nm}$ aluminium film deposited on a silica substrate. A fragment of the SEM image of the sample surface is shown in Fig.1(a). A coherent laser source of $500 \mathrm{~nm}$ illuminated the sample surface from the substrate side. Optical diffraction patterns at different heights to the array were imaged by a digital camera through a combined optical system composed of a high numerical aperture objective lens (N.A. $0.95,150 \times$ ) working in air and another lens with a magnification of 10. Fig.1(b) shows the photograph of part of the sample surface, indicating the full width at 
half maximum (FWHM) of one individual hole is $\sim 400 \mathrm{~nm}$ which is in agreement with the Rayleigh resolution ability of the optical instrument. The diffraction pattern evolved dramatically as distance to the array varied. However, at some distances we observed subwavelength structures, Fig.1(c-d). The sizes of the small dots are considerably smaller than that of an individual hole shown in Fig.1(b). The FWHM of the central small spot in Fig.1(c) is $\sim 240 \mathrm{~nm}(0.48 \lambda)$ and that highlighted in Fig.1(d) is $\sim 170 \mathrm{~nm}(0.34 \lambda)$, which are much smaller than the Rayleigh resolution of the microscope $(320 \mathrm{~nm}, 0.64 \lambda)$. The subwavelength structures were imaged with a conventional optical microscope in the far field, thus had no contribution from evanescent fields.

To confirm that the quasi-periodic nanohole array is able to create subwavelength spots without evanescent waves, we performed simulations of the diffraction pattern based on the angular spectrum representation of the diffracted field. To compute the diffraction patterns with 14,000 holes will require a huge amount of computation time. As a proof-of-concept, we did simulations with 530 holes and took into account only the propagating waves with spatial frequencies smaller than the wavevector of light $(\lambda=500 \mathrm{~nm})$. Fig.1(e) shows the calculated diffraction pattern at a distance of $h=7.1 \mu \mathrm{m}$, which strongly resembles the image shown in Fig.1(c). Subwavelength spots are evident, e.g., the central spot in the image indicates a FWHM of $160 \mathrm{~nm}(0.32 \lambda)$ which is well below the Rayleigh resolution of an optical lens.

The nanohole array was also found to form well-isolated hot spots of electromagnetic energy concentration in free space. Fig.2(a) shows a diffraction pattern measured by a SNOM probe (aperture $\sim 120 \mathrm{~nm}$ ) at a distance of $h=12.5$ $\mu \mathrm{m}$ when illuminated by a diode laser of $660 \mathrm{~nm}$. Hot spots isolated a few microns away from neighboring dots are sparsely distributed in the image plane. The image of a single hot spot marked in image (a) is zoomed in Fig.2(b), which clearly shows a well-defined elliptical dot of subwavelength size. Line profiles along the short axis and long axis of the dot are shown in Fig.2(c), which indicates a FWHM along the short axis (blue dots) of $\sim 320 \mathrm{~nm}$ and that along the long axis (red dots) of $\sim 420 \mathrm{~nm}$. After convolution to take into account the size of probe aperture, the actual size of the focal spot is about $400 \times 290 \mathrm{~nm}(0.6 \lambda \times 0.44 \lambda)$. With focused hot-spots of such high localization and brightness, resolution exceeding that of sophisticated high-numerical aperture lenses imaging can be achieved by scanning the object under investigation across the focal spot. Similarly, a single hot-spot appropriately isolated by a mask may be used as a "light pen" in high resolution photo-lithography.
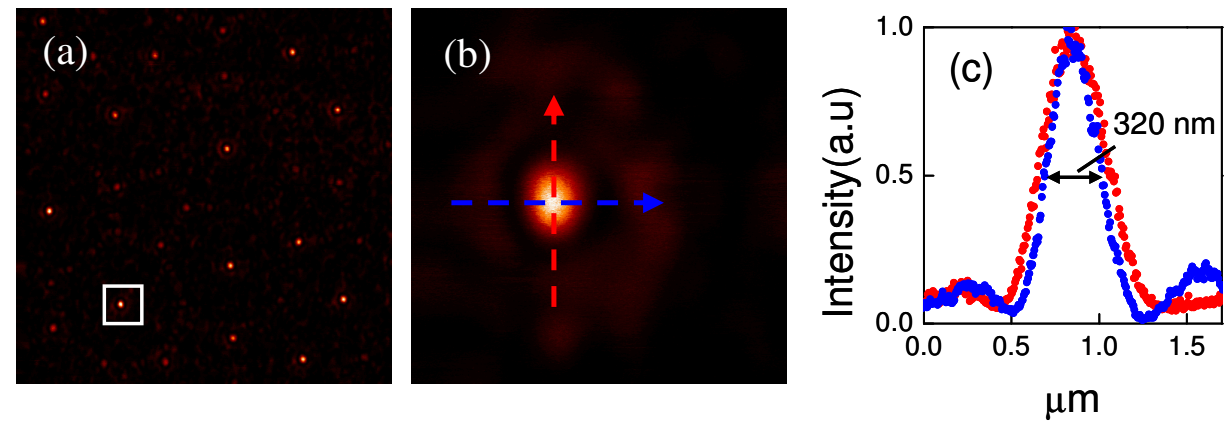

Fig.2 Well-isolated subwavelength hot-spot $(0.6 \lambda \times 0.44 \lambda)$ formed by a quasi-periodic nanohole array. The illumination source is a diode laser with a wavelength of $660 \mathrm{~nm}$. (a) field map $(30 \times 30 \mu \mathrm{m})$ measured at a height $h=12.5 \mu \mathrm{m}$ above the array by a scanning near-field optical microscope; (b) zoomed image ( $3 \times 3 \mu \mathrm{m})$ of a single hot spot highlighted in image (a); (c) profiles of the focus along horizontal (blue dots) and vertical (red dots) directions, indicating a FWHM of $\sim 320 \mathrm{~nm}$ along the horizontal axis.

In conclusion, we provide the first experimental evidence of subwavelength focusing without evanescent fields using a planar photonic structure. Furthermore, we observed that the quasi-crystal nanohole array produces wellisolated hot spots at distances of tens of microns away from the array, which may find applications in subwavelength imaging or high resolution photolithography.

[1] M. V. Berry and S. Popescu, "Evolution of quantum superoscillations and optical superresolution without evanescent waves", J. Phys. A: Math.Gen. 39, 6965-6977 (2006). 